\title{
(2) OPEN ACCESS \\ Evaluation of a clinical pharmacist consultation service for patients with infectious diseases in China: a systematic review and meta-analysis
}

\author{
Jiaxing Zhang, ${ }^{1}$ Xiaosi Li, ${ }^{2}$ Juan Xie, ${ }^{1}$ Wenyi Zheng ${ }^{3}$
}

\begin{abstract}
- Additional material is published online only. To view please visit the journal online (http://dx.doi.org/ 10.1136ejhpharm-2018001815).
\end{abstract}

${ }^{1}$ Department of Pharmacy, Guizhou Provincial People's Hospital, Guiyang, China ${ }^{2}$ Department of Pharmacy, Hospital of Chengdu Office of People's Government of Tibetan Autonomous Region, Chengdu, China

${ }^{3}$ Experimental Cancer Medicine, Clinical Research Center, Department of Laboratory Medicine, Karolinska Institute, Stockholm, Sweden

\section{Correspondence to}

Dr Xiaosi Li, Department of Pharmacy, Hospital of Chengdu Office of People's Government of Tibetan Autonomous Region, Chengdu 610000, China; lixiaosiyyy@126.com

Received 20 November 2018 Revised 13 May 2019

Accepted 30 May 2019 Published Online First 20 June 2019

EAHP Statement 4: Clinical Pharmacy Services.
D) Check for updates

(C) European Association of Hospital Pharmacists 2020. Re-use permitted under CC BY-NC. No commercial re-use. Published by BMJ.

To cite: Zhang J, Li X, Xie J, et al. Eur J Hosp Pharm 2020;27:131-136.

\begin{abstract}
Background Since antibiotic resistance has become a global public health issue, a clinical pharmacist consultation service has been established in China.

Objectives To clarify the role of the clinical pharmacist consultation service in the management of infectious diseases (ID).

Methods Medline, Embase, Cochrane Library, Chinese National Knowledge Infrastructure, Chinese Biomedical Database, Vip Database and Wanfang Database were searched for studies meeting predefined inclusion criteria. The pooled acceptance rate of consultation (ARC) and effective rate of patients treated with the acceptance of pharmacists' suggestions (ERA) were then calculated.

Results Fifty studies (involving 8616 patients and 8828 consultations) from 17 different provinces in China were included. The pooled ARC and ERA were $93.13 \%$ (95\% Cl 90.62\% to $95.00 \%)$ and $93.60 \%(95 \% \mathrm{Cl}$ $91.99 \%$ to $95.24 \%$ ), respectively. Adopting pharmacists' suggestions can significantly improve the prognosis of patients with ID with a risk ratio of $2.08(95 \% \mathrm{Cl} 1.41$ to 3.06). Factors such as study time, characteristics of the research institution and patients have important influences on ARC.

Conclusion Prospective cohort studies with better methodology are needed, although a series of uncontrolled studies has shown that the service has excellent acceptance and improves the effectiveness of treatment for ID.
\end{abstract}

\section{INTRODUCTION}

Clinical pharmacy ${ }^{1}$ refers to the discipline in which the pharmacist provides patient care that optimises medication therapy and promotes health, wellness and disease prevention. Clinical pharmacists work closely with both clinicians and patients to ensure that the medications prescribed for patients contribute to the best possible health outcomes.

Nevertheless, the development of hospital clinical pharmacy in China is far behind that in developed countries. For medical institutions of the same scale (1000 beds), the number of pharmacists in the Johns Hopkins Hospital (0.04 per bed) is eight times that in a general tertiary hospital in China (0.005 per bed). ${ }^{2}$ The shortage of human resources in China results in low accessibility to healthcare from clinical pharmacists. The situation is, however, improving as a result of implementation of a clinical pharmacist consultation service, which generally consists of four steps: (1) clinicians send a consultation request to the Department of Clinical Pharmacy when treating patients with complicated diseases; (2) the Department of Clinical Pharmacy assigns the pharmacists with specialist board certifications through 1 year residency training to deal with the consultation; (3) the designated pharmacists reply with treatment suggestions (eg, selection of antibiotics) based on the benefits and risks of medications; (4) the clinicians make the final decision on the therapeutic scheme.

The severity of infectious diseases (ID) in hospital is increasing due to an increase in drugresistant bacteria and abuse of antibiotics. ${ }^{3-5}$ Infections caused by resistant pathogens are difficult to treat and are associated with increased morbidity, mortality and costs. ${ }^{67}$ The United States Centers for Diseases Control and Prevention estimates that antibiotic-resistant pathogens cause two million infections and 23000 deaths each year in the USA. ${ }^{8}$ In China, the resistance of clinical isolates is also widespread with a continuing upward tendency. ${ }^{9}$ In response to the increasingly severe situation, the Ministry of Health of China launched the Antimicrobial Rectification Action ${ }^{10}$ in 2011 and successively promulgated a series of antimicrobial stewardship regulations requiring pharmacists to participate in the management of antimicrobial drugs. Pharmacists are gradually being incorporated into the consortium for treatment of infectious patients via the consultation service, and there are accumulating studies focusing on the role of pharmacists in antiinfection consultation in China. However, these studies are case series reporting consultations in individual institutions and the results vary greatly. A study summarising the acceptance of the consultation service at a nationwide level is not available.

We conducted a systematic review to investigate the present status of the pharmacist consultation service in China and to evaluate the effectiveness of this service for patients with ID at a nationwide level.

\section{METHODS}

\section{Literature search}

We searched PubMed, EMBASE, the Cochrane Central Register of Controlled Trials (CENTRAL) published in the Cochrane Library using the search strategy detailed in online supplementary table S1; we also searched Chinese National Knowledge Infrastructure (CNKI), Chinese Biomedical Database (CBD), Vip Database (VD) and Wanfang Database (WD) for literature published in Chinese and the electronic databases of the Chinese Pharmaceutical Association (CPA). The references of all retrieved articles were checked for additional 


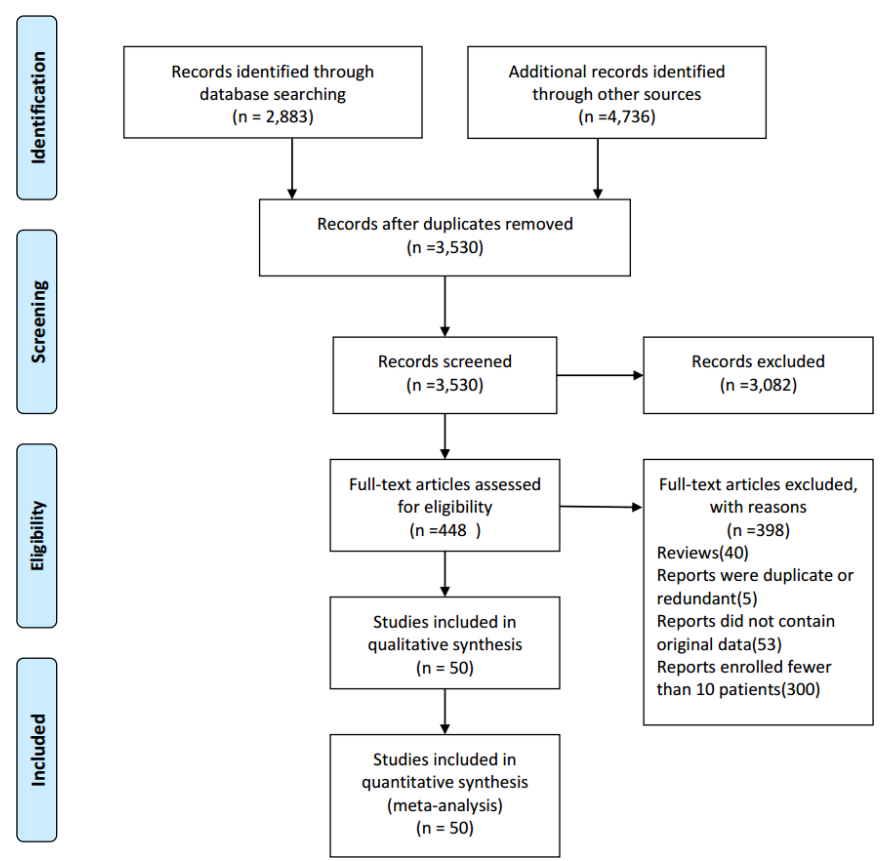

Figure 1 Modified four-phase PRISMA 2009 flow diagram that maps out the number of records identified, included and excluded, and the reasons for exclusions.

relevant publications. We searched all databases from their earliest records to May 2018.

\section{Eligibility criteria}

All included studies met the following criteria: (1) case series enrolling more than 10 patients; (2) inpatients (including both children and adults) with serious infections in a Chinese hospital and in need of pharmacist consultation; (3) intervention of pharmacist consultation; and (4) information on at least one of the following outcomes.

The primary outcome was the acceptance rate of consultation (ARC) measured by comparing the prescriptions of clinicians with the suggestions of pharmacists. Acceptance indicates that the clinicians completely or partially adopted the advice of pharmacists, and ARC was the proportion of consultation suggestions adopted by clinicians to the total consultation requests sent by clinicians.

The secondary outcome was effective rate (ER) evaluated according to the 'Guiding principles for clinical application of antibiotics' (2004). ${ }^{11}$ Effective response is defined as healing, improvement, free of symptoms with bacterial colonisation or discharged after recovery. Healing means recovery of symptoms, signs, laboratory test results and pathogenic test results, while improvement represents obvious remission but with one of the above indicators not completely recovered. ER was the proportion of patients achieving an effective response.

\section{Study selection and data extraction}

Two authors (JZ and XL) independently screened the titles and abstracts of all studies identified by the search strategy and assessed the studies for inclusion using predetermined inclusion criteria. The full texts of all potentially relevant articles were retrieved for detailed review. We resolved disagreements by discussion until consensus was achieved.

Two authors (JZ and XL) used a pre-designed data collection form to independently extract data from each included study. The following data were extracted: (1) patient characteristics including number of patients meeting the inclusion criteria, number of consultation applications (multiple rounds of consultation for one patient), gender, age and the type of infectious bacteria; (2) study time and design; (3) information on the research institution including geographical location, rank graded by the Hospital Classification Management Standard and region according to economic level; (4) number of consultation suggestions adopted by clinicians, number of responsive patients whose treatment adopted pharmacists' suggestions or not, and total number of patients whose treatment adopted pharmacists' suggestions or not.

\section{Quality assessment}

Quality assessment was based on the checklist for assessment of case series developed by the UK National Institute for Health and Care Excellence (NICE). ${ }^{12}$ We answered eight questions in the checklist with yes, no, or unclear.

\section{Statistical analysis}

Studies from the same institution conducted at different times were integrated into one study before analysis. We achieved exact binomial 95\% CI of ARC and ER in each study. We estimated the between-study variance and determined pooled estimates of ARC and ER using the software R 3.3.1 with a random-effect model. ${ }^{13}$ For dichotomous outcomes, if the primary studies reported the relative risks (RR), the pooled RR with $95 \% \mathrm{CI}$ was calculated using RevMan 5.1 with a random-effect model. Heterogeneity among studies was assessed using the Cochran's Q-statistic and $\mathrm{I}^{2}$ test. Significant heterogeneity was indicated if the corresponding $\mathrm{p}$ value of the Cochrane's $\mathrm{Q}$ test was $<0.01$ or $\mathrm{I}^{2}>50 \%$. Considering possible confounders (eg, study time, characteristics of research institution and patients), subgroup analysis was also performed. Publication bias was assessed with the funnel Egger test in R 3.3.1, and $p \leq 0.05$ suggested a significant publication bias. ${ }^{14}$ Sensitivity analysis was performed using software R 3.3.1.

The kappa statistic $(\kappa)$ was calculated for measuring agreement between two authors on the decisions made in study selection. $\mathrm{K}$ values of $0.40-0.59,0.60-0.74$ and $>0.75$ represent fair, good and excellent agreement, respectively. ${ }^{15}$ The design and report of this review was checked with the PRISMA checklist.

\section{Data availability}

All the data generated or analysed during this study were included in this article (and the online supplementary information files).

\section{RESULTS}

\section{Study selection}

A total of 3530 publications were identified from the literature search and $50^{16-65}$ (8616 patients and 8828 consultations) were eligible and are included in this systematic review (figure 1). Agreement between the two reviewers for study selection was excellent $(\kappa=0.80)$. As shown in online supplementary table $S 2$, all the included studies were case series published in Chinese and available as full text. The study time ranged from Febuary 2005 to December 2014, with 12 studies before 2011.

\section{Institutions and patients}

The institutions consisted of 36 tertiary hospitals and 14 secondary hospitals from 17 provinces in China. Most of them were in the eastern region $(n=27)$ while 13 and 10 were in the central and western regions, respectively. The patient characteristics are described in online supplementary table S2. A total of 
Table 1 Results of meta-analysis of ARC

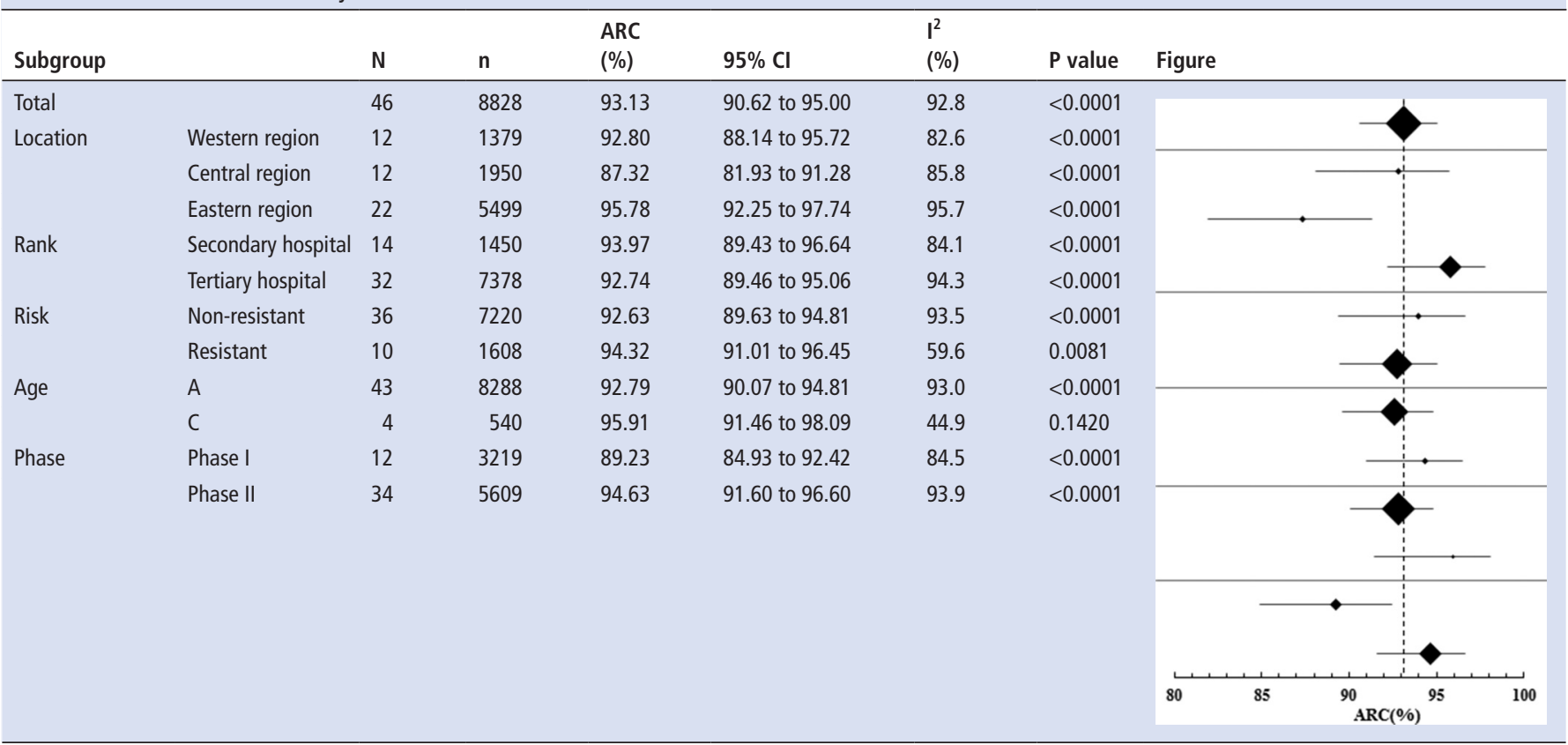

A, adults and children; ARC, acceptance rate of consultation; C, children; N, number of studies; n, number of consultations; non-resistant, non-resistant bacteria infection; resistant, resistant bacteria infection; phase I, study time before 2011 ; phase II, study time after $2011 . \mathrm{I}^{2}$ and $\mathrm{p}$ value, results of heterogeneity test.

1698 patients $(19.71 \%)$ had resistant bacteria infection, and four studies involved a total of 538 children.

\section{Quality assessment}

As shown in online supplementary table S3, every study was conducted at a single centre and all described the hypothesis/ objective and main findings clearly; however, only half of the studies defined the outcomes and patient inclusion criteria. Most studies did not provide information on data collecting; only one collected data prospectively and a further two enrolled patients consecutively. Five studies stratified patients according to the clinical characteristics.

\section{Acceptance rate of consultation (ARC)}

ARC was reported in all studies. After merging seven studies $^{2836-38535462}$ from three identical institutions, 46 studies were subjected to final analysis. The primary data were logittransformed due to non-normal distribution before analysis. Online supplementary figure S1 shows that ARC ranged from $53 \%$ to $100 \%$ with a pooled rate of $93.13 \%$ (95\% CI $90.62 \%$ to $95.00 \%)$. The heterogeneity among studies was statistically significant $\left(\mathrm{I}^{2}=92.8 \%, \mathrm{p}<0.0001\right)$.

To explore the cause of heterogeneity, subgroup analyses were performed according to geographical location, institution rank, risk, age and study time, respectively (table 1). The results indicated that ARC was highly dependent on geographical location with a descending order of eastern (95.78\%),western $(92.80 \%)$ and central regions $(87.32 \%)$. Interestingly, ARC in secondary hospitals was slightly higher than in tertiary hospitals (93.97\% vs 92.74\%). Higher ARC was also found in the context of resistant bacterial infection compared with nonresistant bacterial infection (94.32\% vs $92.63 \%)$, and in children compared with the total population (95.91\% vs $92.79 \%)$. ARC increased by around 5\% after 2011 compared with before 2011 (94.63\% vs $89.23 \%)$. These differences had no statistical significance, although the heterogeneity among studies decreased by subgroup analysis.

\section{Effective rate of patients treated after acceptance of pharmacists' suggestions (ERA)}

After data screening,

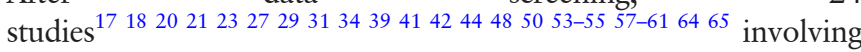
2745 patients were finally included in the analysis. Online supplementary figure S2 shows that the ERA ranged from 69\% to 100\% and the pooled rate was $93.60 \%$ (95\% CI 91.99\% to $95.24 \%$ ). Because of the significant heterogeneity among studies $\left(\mathrm{I}^{2}=66.8 \%\right.$, $\mathrm{p}<0.0001)$, subgroup analyses were performed according to infection risk (table 2). ERA of resistant bacterial infection $(94.43 \%)$ was higher than that of non-resistant bacterial infection (93.34\%), but the difference was not statistically significant.

Table 2 Results of meta-analysis of ERA

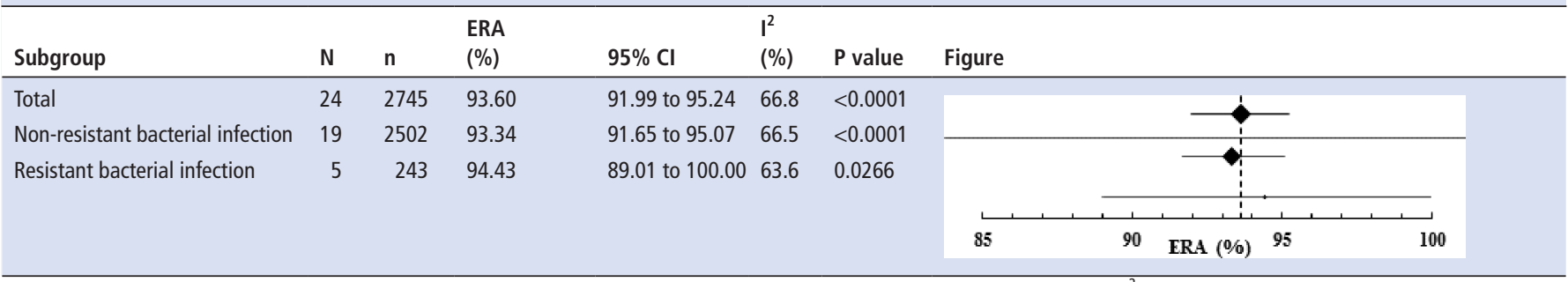




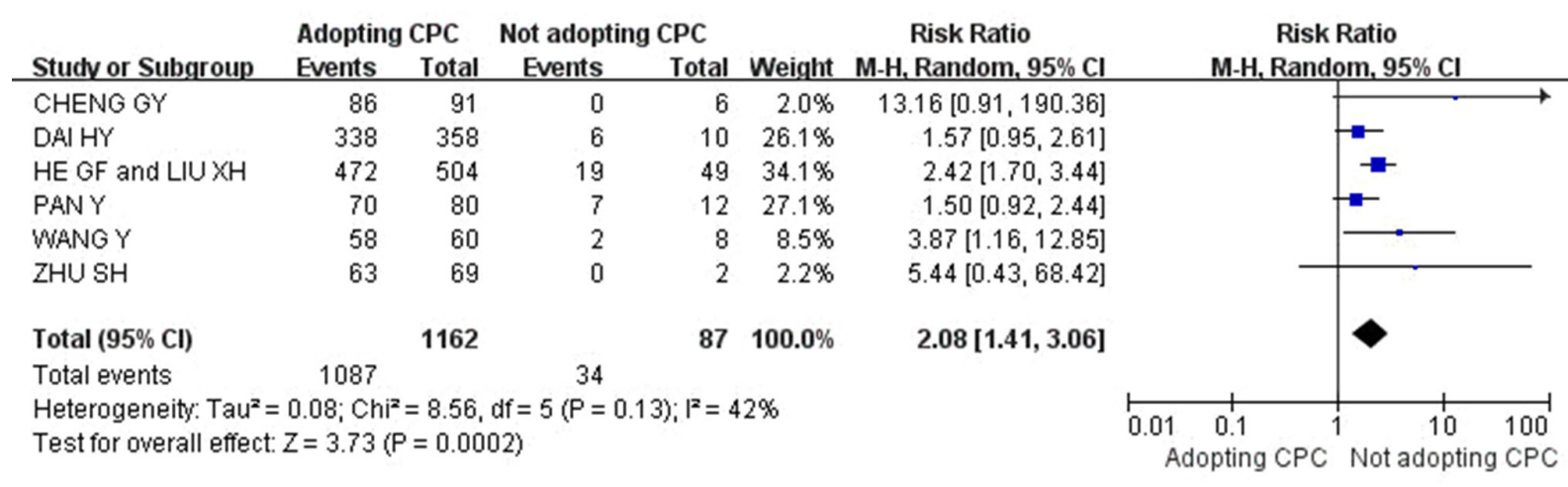

Figure 2 Meta-analysis forest plot of effective rate of patients in the two groups. CPC, clinical pharmacists' consultation opinion.

Among the 24 studies included, six studies $34 \quad 4853-555758$ involving 1249 patients reported the ER of 1162 patients whose treatment followed pharmacists' suggestions and 87 whose treatment did not follow the pharmacists' suggestions. To compare the ER between the two groups, a meta-analysis using RR with 95\% CI was conducted with a random-effect model. As shown in figure 2, the pooled RR was 2.08 (95\% CI 1.41 to 3.06, $\mathrm{p}=0.0002$ ), meaning that adoption of pharmacists' suggestions could significantly improve the prognosis of patients with ID. The heterogeneity among studies was not statistically significant $\left(\mathrm{I}^{2}=42 \%, \mathrm{p}=0.13\right)$.

\section{Publication bias}

The funnel plot was graphed and the Egger test was conducted to investigate publication bias in the 46 studies reporting ARC and 24 studies reporting ERA in patients with ID. As shown in online supplementary figures S3 and S4, both the asymmetrical funnel plots and the results of the Egger test $(p=0.003193$ and $p=0.002872$, respectively) demonstrated the presence of publication bias. Most studies reported a higher ARC, especially those with small sample size.

\section{DISCUSSION}

Fifty studies from 17 different provinces were included for analysis and we take these to be representative of the general status in China. According to the results of the meta-analysis, the consultation service for ID is well accepted by clinicians in China with a high ARC of 93.13\%, and improves the effectiveness of treatment for ID with an excellent ERA of 93.60\% (95\% CI $91.99 \%$ to $95.24 \%$ ) and RR of 2.08 (95\% CI 1.41 to 3.06 ).

To fight against growing antimicrobial resistance, many countries have implemented an antimicrobial stewardship programme (ASP). ${ }^{66}$ Pharmacists' contributions to ASP have been proved to be essential. The inclusion of pharmacists in the ASP has resulted in a significant improvement in the quality of antibiotic use in both developing $^{67-70}$ and developed ${ }^{71-78}$ nations. In China, due to the insufficient number of clinical pharmacists and the regulation that the use of some special antibiotics (eg, vancomycin, carbapenem and tigecycline) needs to be reviewed and approved by clinical pharmacists (Measures for the Administration of Clinical Use of Antibacterials), a pharmacist-led consultation service became a major way for pharmacists to participate in the treatment of ID. During the process of consultation, clinical pharmacists participate in the treatment of ID through a variety of ways (eg, round with clinicians, discussion with patients, link with microbiologists and nurses, developing a therapeutic scheme, adjusting the dosage regimen according to pharmacokinetic-pharmacodynamic results and monitoring adverse events). The multiple contributions of clinical pharmacists can help clinicians to optimise the treatment and improve patient outcome, which may explain the high scores of ARC and ERA.

According to the subgroup analysis, multiple factors (location, rank of research institution, study time, patient characteristics) have important effects on ARC. The increasing ARC after 2011 might result from implementation of the Antimicrobial Rectification Action which permits clinical pharmacists to participate more actively in the healthcare team. Higher ARC in the western region and in secondary hospital implies that clinicians from institutions of a lower economic and medical level are more prone to collaborate with the pharmacists. In addition, the clinicians incline to adopting the pharmacists' suggestions when treating patients with complicated conditions (eg, children or resistant bacterial infections). The limited sample size may result in the insignificant differences among subgroups.

There are some limitations to our analysis. First, the $\mathrm{I}^{2}$ value reported in the meta-analysis $\left(\mathrm{I}^{2}=92.8 \%\right)$ for $\mathrm{ARC}$ is very high. Although we conducted the meta-analysis using a random-effect model and then performed subgroup analyses to explore the cause of heterogeneity, most $\mathrm{I}^{2}$ values remain over $85 \%$, which implies the presence of other confounders such as the professionalism of the clinical pharmacist, disease severity, and financial as well as psychological factors affecting the physician's decision. Unfortunately, none of the included studies provided this information so further analyses of heterogeneity were not conducted. However, sensitivity analyses by successively omitting studies (Figures S5 and S6) demonstrated a high level of robustness of the results from the meta-analyses. Furthermore, most of the studies eligible for ER analysis in this review failed to compare the effectiveness between adopting pharmacists' suggestions and not. Also, the obtained ER may be influenced by many confounders such as severity of infection, cause and development of ID. Studies on this topic with a better methodological design such as prospective cohort studies or controlled studies are urgently needed. Consecutive enrolling of patients can help to reduce selection bias in prospective multicentre case series studies; however, few studies provided information on this, which needs improvement in future studies. In addition, publication bias still exists, although we included those studies enrolling 10 or more patients to avoid extreme risk of reporting and selection bias from smaller studies. The results therefore need to be verified in large-sample studies with registered protocols.

Recently, increasing evidence has proved the positive impact of the clinical pharmacy service on disease treatment, especially for chronic diseases. Zhong et $a l^{79}$ conducted a 
systematic review including eight randomised controlled trials with 1327 patients and clarified the impact of pharmacist care for outpatients with chronic obstructive pulmonary disease. Although current evidence failed to illustrate a significant improvement in the health-related quality of life in intervention patients, pharmacist care improved medication compliance (RR $1.23,95 \%$ CI 1.11 to 1.36 ) and reduced hospital admissions (RR $0.50,95 \%$ CI 0.39 to 0.64 ) and health-related costs (standardised mean difference -0.37 , $95 \%$ CI -0.59 to -0.15$)$. For ID, pharmacists have to deal with every consultation request according to the requirement of the Antibiotic Clinical Research Guiding Principle. Considering the ethics and feasibility, it is impossible to perform trials to assess the effectiveness of the consultation service for ID so the studies included in our review are all observational. Peng et $a l^{80}$ performed a literature analysis of 148 references (including a case report with a small sample $(\mathrm{n}<10))$ on pharmacy consultation in China. In line with the findings in our study, they noted that the pharmacy consultation service in China was poor before 2010 but improved greatly due to the support and recognition of the management staff and clinicians. However, the study by Peng et al ${ }^{80}$ is a qualitative description and lacks evaluation of research quality and quantitative analysis. In our study we assessed the quality of the included studies and also investigated the present research status of the pharmacist consultation service for ID by meta-analysis and subgroup analyses, calculated the pooled ARC and ERA and determined the important factors associated with the ARC.

\section{CONCLUSION}

The consultation service has provided a significant breakthrough for clinical pharmacy in China and has achieved some success. Our study shows that the pharmacist-led consultation service may be an effective intervention for ID treatment, which can be shared with other developing countries where clinical pharmacists are not actively involved in patient healthcare. To the best of our knowledge, this is the first systematic review investigating the role of a pharmacist consultation service in ID, which is believed to shed light on this service in China. Because of the limitations of the included studies, our group is planning to establish a follow-up registry database and to conduct a prospective cohort study based on our clinical pharmacist consultation system in the western region of China to further evaluate the effectiveness of pharmacists' consultation for ID and investigate the important associated factors.

Acknowledgements We thank Professor Jing Li and Professor Deying Kang from the Chinese Cochrane Center in West China Hospital of Sichuan University for providing methodology and statistical support for this review.

Contributors $\mathrm{JZ}, \mathrm{XL}$ and $\mathrm{JX}$ conceived and designed the experiments. JZ, XL and WZ performed the experiments. JZ, XL and WZ analysed the data. JZ, XL and JX contributed reagents/materials/analysis tools. JZ, XL, WZ and JX wrote the paper.

Funding This study was supported by Beijing Medical and Health Foundation, grant number YWJKJJHKYJJ-B17444.

Competing interests None declared.

Patient consent for publication Not required.

Provenance and peer review Not commissioned; externally peer reviewed.

Open access This is an open access article distributed in accordance with the Creative Commons Attribution Non Commercial (CC BY-NC 4.0) license, which permits others to distribute, remix, adapt, build upon this work non-commercially, and license their derivative works on different terms, provided the original work is properly cited, an indication of whether changes were made, and the use is noncommercial. See: http://creativecommons.org/licenses/by-nc/4.0/.

\section{REFERENCES}

1 American College of Clinical Pharmacy. The definition of clinical pharmacy. Pharmacotherapy 2008;28:816-7.

2 Zhen JC. Pharmaceutical research project in Johns Hopkins University in 2015 (study report from Beijing group). Available: http:/www.clinphar.cn/thread-329496-1-1.htm

3 Meek RW, Vyas H, Piddock LJV. Nonmedical uses of antibiotics: time to restrict their use? PLoS Biol 2015;13:e1002266.

4 Vincent J-L, Rello J, Marshall J, et al. International study of the prevalence and outcomes of infection in intensive care units. JAMA 2009;302:2323-9.

5 Hidron Al, Edwards JR, Patel J, et al. Antimicrobial-resistant pathogens associated with healthcare-associated infections: annual summary of data reported to the National Healthcare Safety Network at the Centers for Disease Control and Prevention, 2006-2007. Infect Control Hosp Epidemiol 2008;29:996-1011.

6 Schwaber MJ, Carmeli Y. The effect of antimicrobial resistance on patient outcomes: importance of proper evaluation of appropriate therapy. Crit Care 2009;13:106-28.

7 Giske CG, Monnet DL, Cars O, et al. Clinical and economic impact of common multidrug-resistant gram-negative bacilli. Antimicrob Agents Chemother 2008:52:813-21.

8 Centers for Disease Control and Prevention. Antibiotic resistance threats in the United States, 2013. Available: http://www.cdc.gov/drugresistance/threat-report-2013/ [Accessed 20 Feb 2016].

9 Xiao YH, Shen P, Wei ZQ, et al. Mohnarin report of 2010: Surveillance of bacterial resistance in China. Chin J Nosocomiol 2011;21:4896-902.

10 Ministry of Health of China. Notice of the general office of the Ministry of Health on the special rectification activities of clinical application of antibacterial drugs in China. National Health and Family Planning Commission of People's Republic of China. Available: http://www.moh.gov.cn/mohyzs/s3586/201104/51376.shtml

11 Ministry of Health of China. Guiding principles for clinical application of antibiotics. National Health and Family Planning Commission of People's Republic of China. Available: http://www.moh.gov.cn/wsb/pzcjd/200804/21154.shtml

12 National Institute for Health and Clinical Excellence (NICE). Quality assessment for case series. Available: https://www.nice.org.uk/guidance/cg3/documents/appendix-4quality-of-case-series-form2

13 DerSimonian R, Laird N. Meta-analysis in clinical trials. Controlled Clinical Trials 1986:7:177-88.

14 Egger M, Smith GD, Schneider M, et al. Bias in meta-analysis detected by a simple, graphical test. BMJ 1997;315:629-34.

15 Landis JR, Koch GG. The measurement of observer agreement for categorical data. Biometrics 1977:33:159-74.

16 Zhao HX. Analysis of 162 cases of multidrug resistance and pandrug resistant bacterial infection in clinical pharmacists. Anhui Med Pharm J 2014;18:1377-8.

17 Li L, Zhang XF, Zeng YZ, et al. The efficacy of fosfomycin for the treatment of nosocomial infections caused by carbapenem-resistant Klebsiella pneumoniae. Med J West China 2015:27:611-3.

18 Huang YP. Retrospective analysis of clinical consultations for special class antibacteria drugs in a primary hospital. Chin Pharm 2015;24:97-8.

19 Wang HM, Zhu SY, XY L, et al. Analysis on 307 intractable cases consultations with clinical pharmacists participating in our hospital. China Pharmacy 2015;26:1134-6.

20 Liang ZM, Liang BY. Analysis of 79 cases of multi-drug-resistant and pan-drugresistant bacterial infection consulted by clinical pharmacist. China Pharmacy 2011;22:2098-101.

21 Pang TY. Retrospective analysis of 106 clinical use of consultation of specialized antibacterial drugs in our hospital. J North Pharmacy 2014;11:117-8.

22 Cai JY, MX S, Cao K, et al. Clinical analysis of anti-infective cases of clinical pharmacist consultation in our hospital in 2014. J North Pharmacy 2015;12:162-3.

23 Pang XJ, Wei $\mathrm{H}$, Chen Y, et al. Clinical pharmacists involved in the treatment of the drug resistance of Enterobacter cloacae urinary tract infection. Chin Hosp Pharm J 2010;30:701-2.

24 HB M. Clinical analysis of stroke and multiple drug resistance and pan-drug resistant bacterial infection. Modern Journal Integrated Traditional Chinese and Western Medicine 2014:23:3159-60.

25 Fang WJ, Zhang YL, Yuan M. Evaluation on medication regimens of 30 reports of pan-drug resistant gram-negative bacilli infection in our hospital. China Pharmacy 2015;26:1928-30.

26 Tang SY, Liu TT, Jiang $X$, et al. Analysis of clinical pharmacists participating in 300 cases of children antibacterial drugs consultation in our hospital during 2011-2013. China Pharmacy 2014;25:4404-8.

27 Wei YJ. Analysis of clinical pharmacists participating in 51 cases of pediatric infectious diseases. Chin J Clinical Rational Drug Use 2014;7:55-6.

28 Zhang C, Fang XL, Li F, et al. Analyses of consultations supplied by clinical pharmacist for 23 children with urinary tract infection. J Pediatr Pharmacy 2016:22:34-7.

29 Wang HF, Jia P. Clinical pharmacists participating in consultation on 164 cases of pediatric bacterial infectious diseases. Chin Pharm 2014;23:78-9. 
30 Shi GY. 100 cases consultation of anti-infective drugs by clinical pharmacist. World Clin Drug 2014;35:633-6.

31 Qin L, Zhao PX, RR W, et al. Clinical pharmacists' involvement in consultation: analysis of 90 infective cases. Evaluation and Analysis of Drug-Use in Hospitals of China 2012;12:756-9.

32 Wang T. Effect of clinical pharmacists participating in the multidisciplinary consultation in the recent 2 years. Chin Pharm 2015;24:59-60.

33 Lin SZ, Lai SC, XF S. Analysis of 41 clinical pharmacist consultations. Chin Lic Pharm 2014;11:16-19.

34 Zhu SH, Liang YH. Participation of clinical pharmacists in consultation on 71 cases of intractable infectious diseases. Pharmacy Today 2013;23:557-60.

35 Zhu LP, Cheng XL. Experiences of pharmacist participating in clinical consultations. J Pediatr Pharmacy 2012;18:38-40.

36 XQ L, YJ L, RZ L, et al. Clinical pharmacists participating in consultation: analysis of the 317 bacterial infection cases. Chin J Drug App Monitor 2012;9:150-2.

37 QM Y, HFW. Analysis of 127 cases of clinical pharmacists participating in anti infection consultation. Strait Pharm J 2015:27:237-40.

38 YR L, RZ L, Liu RF, et al. Analysis on the clinical pharmacists' participation in the consultation of bacterial infection cases in our hospital during 2010-2013. China Pharmacy 2014;25:4401-4.

39 Xue JW, Huang WZ, Cheng MJ. Analysis of the characteristics and changing trend of bacterial infection cases involving clinical pharmacists. The Chinese and Foreign Health Abstract 2013;35:278-9.

40 Huang YF. Clinical pharmacists participating in consultation for antimicrobial uses of 55 patients. Practical Pharmacy and Clinical Remedies 2012;15:359-60.

41 Yang LH. Case analysis of clinical pharmacist consultation in primary Hospital. Chin J Clinical Rational Drug Use 2015;8:180-1.

42 Han MZ, Yan JH, Liu HY. Analysis of 63 cases of clinical pharmacists participating in the consultations. Chongqing Med 2012;41:3754-6.

43 Li M, Geng WF, Yin H. Analysis on clinical pharmacists participating in anti-infection treatment consultation in wards. J Guiyang Med Coll 2015;40:205-7.

44 TY L, Chen MY, Chen H, et al. Analysis of clinical pharmacists participating in 91 cases of clinical consultation. China Pharmacy 2014;25:2868-70.

45 Gao YP, Zhang JM. Retrospective analysis of antimicrobial drugs consultation for clinical pharmacist. China Modern Medicine 2012;19:139-41.

46 Song J. Investigation of clinical pharmacist participating in consultation about infectious diseases in our hospital. China Modern Doctor 2012:50:123-4.

47 LM X, Xue GY. Clinical pharmacist-involved consultation records in 2012 and 2013: a contrast analysis. Evaluation and Analysis of Drug-Use in Hospitals of China 2014;14:566-8.

48 Cheng GY, JM H, Feng YG. Analysis of clinical pharmacists participating in 97 cases of infectious disease consultation. China Pharmacist 2012;15:96-8.

49 Zhang Q, Zhen JH. Analysis of 251 cases with infectious diseases. Chin Hosp Pharm J 2011:31:425-6.

50 Qin Y. Practice and experience of clinical pharmacists participating in clinical consultation in 85 cases in our hospital. China Foreign Medical Treatment 2011;11.

51 Zeng JG. Analysis of 22 cases of clinical pharmacist consultations. Chin Pharm $2011 ; 20$

52 DP L. Susceptibility testing of clinical pharmacists in the value of use of antimicrobial drugs. Medical Information 2011;24.

53 GF H, Yuan TL, Zhang SZ, et al. Analysis of 439 cases of infection diseases from neurology ward consulted by clinical pharmacists. China Pharmacist 2015;18:624-7.

54 Liu XH, Zhang SZ, Yang Z, et al. Summary and experiences of anti-infective clinical pharmacist participating in the consultations of 123 cases. Chin J Pharmacoepidemiol 2015;24:436-9.

55 Pan Y, GF H, Liao DH. Consultation analysis of clinical pharmacist on the hospital infection of patients with cancer. Anti-tumor Pharmacy 2015:5:151-5.

56 Yu F. Discussion and experiences on 61 cases of antibiotic therapy in respiratory department which clinical pharmacists participate in. J North Pharmacy 2012;9:77-8.

57 Dai YH, HJ J. Retrospective analysis of 368 clinical pharmacists participating in clinical consultation. J Frontiers of Medicine 2012;2:378-9.
58 Wang Y, Zhang XH, Li J, et al. Analysis of clinical pharmacists participating in 68 cases of infectious diseases in department of neurosurgery. Chin Pharm 2014;23:76-7.

59 Hou GX, Deng J, Ren TS, et al. A retrospective analysis of clinical consultation that clinical pharmacists participate in from 2009 to 2012. Chin J New Drugs Clin Rem 2014:33:545-7.

60 Xie CJ. Analysis of clinical pharmacists participating in consultation infection cases. Jin Ri Jian Kang 2015;14.

61 XF F, Wang QH, SS W, et al. Clinical pharmacist participating in consultation and analysis of 72 cases of antibacterial therapy. Chin Lic Pharm 2014:11:3-6.

62 LX L, Zhang JL, Chen L. Analysis of 292 pharmacy consultation cases with postoperative infection. China Pharmacist 2014;17:116-9.

63 Guan ZS, Zhou XT. Perspective on clinical pharmacists participating in consultation on the use of antibiotics in 227 cases. China Pharmacy 2009:20:1358-60.

64 ZY L, QH H. Participation of clinical pharmacists in consultation on 158 cases of infectious diseases. Central South Pharmacy 2011;9:552-4.

65 Yu M. Analysis of clinical pharmacists engaged in consultation of infectious diseases. Chin Heal Ind 2014;25:143-4.

66 Charani E, Smith I, Skodvin B, et al. Investigating the cultural and contextual determinants of antimicrobial stewardship programmes across low-, middle- and high-income countries—a qualitative study. PLoS One;14:e0209847.

67 Magedanz L, Silliprandi EM, dos Santos RP. Impact of the pharmacist on a multidisciplinary team in an antimicrobial stewardship program: a quasi-experimental study. Int J Clin Pharm 2012;34:290-4.

68 Brink AJ, Messina AP, Feldman C, et al. Antimicrobial stewardship across 47 South African hospitals: an implementation study. Lancet Infectious Diseases 2016:16:1017-25.

69 Zhou Y, Ma L-Y, Zhao X, et al. Impact of pharmacist intervention on antibiotic use and prophylactic antibiotic use in urology clean operations. I Clin Pharm Ther 2015;40:404-8. 35.

70 Shen J, Sun Q, Zhou X, et al. Pharmacist interventions on antibiotic use in inpatients with respiratory tract infections in a chinese hospital. Int I Clin Pharm 2011:33:929-33.

71 Booth JL, Mullen AB, Thomson DAM, et al. Antibiotic treatment of urinary tract infection by community pharmacists: a cross-sectional study. Br I Gen Pract 2013;63:e244-9.

72 Ellis K, Rubal-Peace G, Chang V, et al. Antimicrobial stewardship for a geriatric behavioral health population. Antibiotics 2016;5.

73 Okada N, Fushitani S, Azuma M, et al. Clinical evaluation of pharmacist interventions in patients treated with anti-methicillin-resistant Staphylococcus aureus agents in a hematological ward. Biol Pharm Bull 2016;39:295-300.

74 Northey A, McGuren T, Stupans I. Patients' antibiotic knowledge: a trial assessing the impact of verbal education. Int J Pharm Pract 2015;23:158-60.

75 Yen Y-H, Chen H-Y, Wuan-Jin L, et al. Clinical and economic impact of a pharmacist managed i.v.-to-p.o. conversion service for levofloxacin in Taiwan. CP 2012:50:136-41.

76 Dunn K, O'Reilly A, Silke B, et al. Implementing a pharmacist-led sequential antimicrobial therapy strategy: a controlled before-and-after study. Int I Clin Pharm 2011;33:208-14.

77 Grill E, Weber A, Lohmann S, et al. Effects of pharmaceutical counselling on antimicrobial use in surgical wards: intervention study with historical control group. Pharmacoepidemiol Drug Saf 2011;20:739-46.

78 Hersberger KE, Botomino A, Sarkar R, et al. Prescribed medications and pharmacy interventions for acute respiratory tract infections in Swiss primary care. J Clin Pharm Ther 2009;34:387-95.

79 Zhong H, Ni X-J, Cui M, et al. Evaluation of pharmacist care for patients with chronic obstructive pulmonary disease: a systematic review and meta-analysis. Int I Clin Pharm 2014:36:1230-40.

80 Peng GL, Han Y, Zhang SY, et al. Literature analysis on pharmacy consultation in China. Chin J Hosp Pharm 2016:36:81-4. 